\title{
Dying in the ICU: strategies that may improve end-of-life care
}

\author{
[Mourir à l'USI : les stratégies qui peuvent améliorer les soins en fin de vie]
}

Deborah Cook MD, ${ }^{\star} \dagger$ Graeme Rocker DM, $\ddagger$ Daren Heyland MD $\$$

Purpose: Since 10 to $20 \%$ of adult patients admitted to the intensive care unit (ICU) in Canada die, addressing the needs of dying critically ill patients is of paramount importance. The purpose of this article is to suggest some strategies to consider to improve the care of patients dying in the ICU.

Source: Data sources were randomized clinical trials, observational studies and surveys. We purposively selected key articles on endof-life care to highlight eight initiatives that have the potential to improve care for dying critically ill patients. These initiatives were presented at the International Consensus Conference on End-ofLife Care in the ICU on April 24-25, 2003 in Brussels, Belgium.

Principal findings: We describe eight strategies that, if adopted, may positively impact on the end-of-life care of critically ill patients: I) promote social change through professional initiatives; 2) legitimize research in end-of-life care; 3) determine what dying patients need; 4) determine what families of dying patients need; 5) initiate quality improvement locally; 6) use quality tools with care; 7) educate future clinicians; and 8) personally engage in end-of-life care. Most of these strategies have not been subjected to rigorous evaluation.

Conclusion: Adoption of some of these strategies we describe may lead to improved end-of-life care in the ICU. Future studies should include more formal evaluation of the efficacy of end-of-life interventions to help us ensure high quality, clinically relevant, culturally adapted care for all dying critically ill patients.

Objectif: Au Canada, comme 10 à $20 \%$ des patients admis à l'unité des soins intensifs (USI) y décèdent, il devient primordial de s'occuper des besoins des grands malades en fin de vie. Le but du présent article est de proposer certaines stratégies pour améliorer les soins de ceux qui meurent à l'USI.

Source : Les données proviennent d'essais cliniques randomisés, d'études observationnelles et d'enquêtes. Nous avons volontairement choisi des articles clés sur les soins aux mourants pour mettre en lumière huit initiatives qui peuvent améliorer la condition des grands malades mourants. Ces initiatives ont été présentées à l'International Consensus Conference on End-of-Life Care in the ICU le 24 avril 2003 à Bruxelles en Belgique.

Constatations principales : Nous décrivons huit stratégies qui, si elles sont adoptées, pourraient avoir un impact positif sur les soins aux mourants : 1) promouvoir un changement social par des initiatives professionnelles ; 2) légitimer la recherche sur les soins aux mourants ; 3) déterminer les besoins des mourants ; 4) déterminer les besoins des familles des mourants ; 5) commencer par améliorer la qualité localement ; 6) utiliser les outils de qualité avec précaution ; 7) former les futurs cliniciens et 8) s'engager personnellement dans les soins de fin de vie. La majorité de ces stratégies n'a pas fait l'objet d'évaluation rigoureuse.

Conclusion : L'adoption de certaines stratégies décrites pourrait améliorer les soins aux mourants à l'USI. D'autres études devraient comporter une évaluation formelle de l'efficacité des interventions aux mourants afin de nous aider à assurer des soins de grande qualité, applicables en clinique et adaptés culturellement à tous les grands malades en fin de vie.

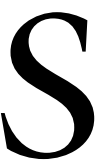

INCE 10 to $20 \%$ of adult patients admitted to the intensive care unit (ICU) in Canada die, addressing the needs of dying critically ill patients is of paramount importance. Recent calls to improve the care of dying patients in the ICU have arisen from several sources including professional societies, hospitals, clinicians, investigators, ethicists, patients, families, and the media. These calls have been made in response to the frequency with which

From the Departments of Medicine, ${ }^{*}$ Clinical Epidemiology and Biostatistics, $†$ McMaster University, Hamilton, Ontario; the Department of Medicine, $\ddagger$ Dalhousie University, Halifax, Nova Scotia; and the Department of Medicine, $\$$ Kingston General Hospital, Kingston, Ontario, Canada.

Address correspondence to: Dr. D.J. Cook, Departments of Medicine and Clinical Epidemiology, McMaster University Medical Center, 1200 Main Street West, Hamilton, Ontario L8N 3A5, Canada. Phone: 905-525-9140, ext. 22900; Fax: 905-524-3841;

E-mail: debcook@mcmaster.ca

Funding: D. Cook is a Canada Research Chair of the Canadian Institutes for Health Research. D. Heyland is a Career Scientist of the Ontario Ministry of Health.

Accepted for publication July 10, 2003.

Revision accepted November 28, 2003. 
critically ill patients receive life support technology, and are perceived to be suffering, or are exposed to interventions that may be inappropriate or unwanted. Most deaths in the ICU are preceded by withdrawal of life support. ${ }^{1}$ Despite these realities, few empiric data exist to guide us concerning provision of optimal endof-life care in the ICU.

Randomized trials outside the ICU have had mixed success in improving the care of seriously ill patients at high risk of death. Ten years ago, the Study to Understand Prognoses and Preferences for Outcomes and Risks of Treatments (SUPPORT) tested a complex information and communication intervention that had no influence on the process or outcomes of care for seriously ill hospitalized patients. ${ }^{2}$ In contrast, simple educational interventions can increase the completion of advance directives, as indicated in a meta-analysis of nine randomized trials. ${ }^{3}$ Inside the ICU, where personal and professional preferences influence the provision or limitation of end-of-life care,${ }^{4,5}$ fewer randomized trials have been performed. One recent trial showed that family members randomized to a family information leaflet had better comprehension, and among those with good comprehension, higher satisfaction with ICU care than family members without the leaflet. ${ }^{6}$ In another randomized trial, an ethics consultation shortened the duration of ICU stay among dying patients without increasing the overall ICU mortality rate. ${ }^{7}$

The dearth of randomized trials in this area notwithstanding, a wide array of interventions has the potential to improve the care of dying critically ill patients. These interventions may be simple, inexpensive and immediate, or complex, costly and implemented over a long period of time. Most of these potentially useful interventions have not been tested formally, and many never will be. However, the lack of proof of their efficacy does not indicate proof of their lack of efficacy.

\section{Source}

Data sources we considered for this report were peer reviewed publications including randomized clinical trials, observational studies and surveys. We purposively selected key articles on end-of-life care in the ICU to highlight eight initiatives that have the potential to improve care for dying critically ill patients. These initiatives were presented by one of us (D.J.C.) at the International Consensus Conference on Endof-Life Care in the ICU on April 24-25, 2003 in Brussels, Belgium.

\section{Main findings}

Herein, we propose eight strategies that, if implemented, may lead to improvements in end-of-life care in the ICU. The ways in which we could have a positive impact on the care of patients dying in the ICU are: 1) promote social change through professional initiatives; 2) legitimize research in end-of-life care; 3 ) determine what dying patients need; 4) determine what families of dying patients need; 5) initiate quality improvement locally; 6) use quality tools with care; 7) educate future clinicians; and 8) personally engage in end-of-life care.

\section{1) Promote social change through professional initiatives}

Many interventions involving professional societies have profoundly affected end-of-life care in the ICU. These interventions represent vehicles for social change, reflected in the creation of new groups, activities and events in the critical care community. For many years, societal documents have shaped our thinking about end-of-life care. ${ }^{8-10}$ In a key publication in the field, Danis and colleagues called for earlier integration of palliative care into critical care. ${ }^{11}$ Recently, the Robert Wood Johnson Foundation promoting excellence in end-of-life care project formed the ICU peer workgroup to galvanize clinical, educational and research activities promoting excellence in end-of-life care for critically ill patients.

Major conferences such as the European Society of Intensive Care Medicine, the International Symposium of Intensive Care and Emergency Medicine, the American Thoracic Society, and the Society of Critical Care Medicine routinely hold symposia devoted to end-of-life care. This topic is likely to stay at the forefront of critical care meetings in years to come. In April 2003, the International Consensus Conference sponsored by the American Thoracic Society, European Respiratory Society, European Society of Intensive Care Medicine, Society of Critical Care Medicine and Societé de Réanimation de Langue Française was devoted to challenges in end-of-life care. This consensus conference symbolizes a unified international commitment to improving the care of dying patients in the ICU. While these initiatives primarily involve physicians, multidisciplinary approaches will be needed to effect optimal social change. In the meantime, an important addition to the bookshelves of many practitioners and trainees next to physiology, monitoring, and therapeutics texts is the recent book by Curtis and Rubenfeld, 'Managing death in the ICU: the transition from cure to comfort'. ${ }^{12}$ 


\section{2) Legitimize research in end-of-life care}

A decade ago, end-of-life research was perceived as less worthy of investigative efforts and funding support than other fields of inquiry in critical care. This image has changed dramatically. In a large, recently launched strategic initiative, the Canadian Institutes for Health Research has committed to palliative and end-of-life care research funding (www.cihrirsc.gc.ca/services/funding/opportunities/institutes $/ 2003 / \mathrm{rfa}$ _8_palliative_care_e.shtml). However, work is still needed at many universities and traditional promotion and tenure committees to ensure that investigative careers in end-of-life care are viewed as legitimate as basic, clinical and health services research directed at other health problems.

The good news is that end-of-life research has grown considerably in quality and quantity, particularly as career scientists have been trained in this field, peer review grants have been obtained, and collaborative research networks have developed. Recently, a multidisciplinary group of practitioners and scientists in the US forged an end-of-life research agenda to help advance the field. ${ }^{13}$ This means that we can look forward to important advances in end-of-life care in the years to come, informed by clinically relevant, culturally adapted research by the ICU community. Canadian investigators have also declared palliative and end-of-life care a research priority. ${ }^{14}$ Future investigations will be best advanced not just by multidisciplinary, but also interdisciplinary, team approaches involving the social and behavioural sciences.

\section{3) Determine what dying patients need}

We may assume that patients dying in the ICU want to have their symptoms well treated, have their wishes respected, and be surrounded by their loved ones. We need to confirm or refute these assumptions by directly approaching critically ill patients, or their loved ones to determine what they need and want from the ICU team. We can begin by drawing useful lessons from other seriously ill dying patients outside of the ICU. To determine the important physician skills for high quality end-of-life care, Curtis and colleagues used 11 focus groups for 79 patients with chronic obstructive pulmonary disease (COPD), cancer and acquired immunodeficiency syndrome (AIDS) in a qualitative study. ${ }^{15}$ Patients stated that emotional support, communication, accessibility and accountability were extremely important. In addition, each group of patients identified factors that were uniquely important to them. The unique theme of cancer patients was maintaining hope despite a terminal disease; that of AIDS patients was pain control. Patients with COPD wanted education about the diagnosis, prognosis, and treatment; they also wanted to know what dying might be like, and to do some advance care planning. While elicited in a study conducted outside the ICU, these universal physician skills (emotional support, communication, accessibility and accountability) may help to improve end-of-life care in the ICU, as they should elsewhere. Similar research on critically ill patients, though difficult, could highlight core competencies for the ICU team. For example, of patients with cancer admitted to a medical ICU in the US, 55 to $75 \%$ of those able to respond reported moderate or severe pain, discomfort, anxiety, sleep disturbance or unsatisfied hunger or thirst, suggesting the need for increased attention to distressing symptoms at the end-of-life. ${ }^{16}$ Other studies suggest that patient recall of the ICU is often unpleasant, underscoring the need to improve symptom control. ${ }^{17}$ In contrast, data from Canadian ICUs are reassuring and suggests that for dying patients, symptom control as perceived by bereaved family members was excellent or very good for the majority of ICU patients. ${ }^{18}$

Valid, reliable, and responsive metrics to measure whether the care of dying patients has improved over recent years are beginning to emerge. One unique tool called the quality of death and dying (QODD) score has been developed and validated in the community setting. ${ }^{19}$ This tool underscores the significant correlations between symptom control and the quality of death. If validated for seriously or critically ill patients, the QODD score could be a prototypical instrument to use for clinical, educational, research and administrative purposes in the ICU.

\section{4) Determine what families of dying patients need}

Caring for dying critically ill patients necessarily means caring for their families. Families of patients in the ICU report being anxious and depressed regarding the illness of their loved one, suggesting considerable vicarious suffering. ${ }^{20,21}$ While these experiences may be minimized by some interventions, they may not be completely eliminated.

To determine what families need from the ICU team regarding decision making, we conducted a multicentre cohort study. ${ }^{22}$ We distributed a validated, self-administered family satisfaction questionnaire assessing 21 key aspects of communication and decision making to 1,123 family members of ICU patients who were mechanically ventilated for at least $48 \mathrm{hr} ; 789$ (70\%) were returned. Families were most satisfied with the frequency of communication with nurses (rated as excellent by $59 \%$ ) and least satisfied with the frequency of communication with physicians (rated as excellent by 
$37 \%)$. In terms of their overall experience regarding decision-making, $71 \%$ were either completely or very satisfied. With respect to their specific role in treatment decisions, $15 \%$ preferred that the physician primarily make the decision, $24 \%$ preferred that the physician make the final decision after considering their opinion, $39 \%$ preferred that the physician shared responsibility to make the decision, $22 \%$ preferred to make the final decision after considering the physician's opinion, and $1 \%$ preferred to make the treatment decision alone. ${ }^{23}$ Adequate communication, feeling supported, and achieving the appropriate level of care for their family member were key determinants of satisfaction with decision-making. Of course, attending to these issues is equally important for family members of survivors and non-survivors of critical illness.

In contrast to the predominantly western view that patient autonomy drives a strong desire for patient decisional authority (and by extension, family decisional authority for non-communicative ICU patients), this study shows that a spectrum of preferences exists for decision-making. Another key observation is that, at least in these participating Canadian ICUs, family members distinguish between information exchange (which most desire) and decision making (which most want to be shared between families and physicians). An important way to improve the care of dying patients in the ICU is to try to understand family members with respect to both their information needs and wishes for involvement in decision-making.

\section{5) Initiate quality improvement locally}

Quality improvement initiatives regarding end-of-life care in the ICU may develop spontaneously or follow a sentinel event. For example in Halifax, Nova Scotia in the aftermath of first degree murder charges laid against an ICU physician regarding treatment of a dying patient (later dismissed), a retrospective review of all deaths in two university affiliated ICUs was conducted. ${ }^{24}$ Large differences were found in technologic and pharmacologic support, drug prescribing, and documentation of physician-family interactions. More important than documenting this practice variation was the consequent institutional support for such initiatives to improve end-of-life care in the ICU.

After the findings of the retrospective review were shared with the ICU team, concerns regarding endof-life care were elicited from nurses and patients. These concerns included perceptions of discomfort during the dying process, insufficient involvement of pastoral care, and suboptimal communication between physicians and others when life support withdrawal was discussed and implemented. Accordingly, policies were developed to address these issues, such as more comprehensive recording of dyspnea, pain, agitation, and their management. Use of open-ended orders with no fixed upper limit was proscribed. A do not resuscitate (DNR) order protocol was created to help clarify resuscitation preferences. A withdrawal of life support checklist was also developed to prompt clinicians to consult with referring physicians, to obtain a second physician's opinion about life support withdrawal, to discontinue technologic interventions incongruent with palliation, to document patient and family discussions with the entire ICU team, and to offer spiritual care to the family. ${ }^{25}$

Local quality improvement initiatives such as this can be powerful; they may not only raise the consciousness of ICU team members about how care can be improved, but they can also actually effect change. Not all of these initiatives may be feasible or suitable for all settings; for example, obtaining second opinions about withdrawal of life support may be best suited for situations in which anguish, anger or discord is either predicted or present among the ICU team and patients or families. Evidence that multimethod initiatives such as this can change behaviour is robust, but the process is complicated, and the success rate in reality is variable. ${ }^{26}$ The foregoing example used five effective behaviour change strategies, including audit (e.g., the published survey of practice patterns), feedback about the audit results (e.g., to the nurses in focus groups), reminders to all ICU clinicians (e.g., the withdrawal of life support checklist), protocols (e.g., the DNR order protocol), and committed opinion leaders (to implement these initiatives). Changing the complex organization of the ICU through local quality improvement initiatives such as this affords many leadership opportunities for all members of the ICU team.

\section{6) Use policy tools with care}

Diverse stakeholders (including clinicians, researchers, patient advocacy groups, lawyers, and ethicists) have attempted to standardize approaches to life-support decisions in the ICU. A plethora of policy tools are now available for this purpose. These include use of advance directives, distribution of professional position papers, protocols, and bedside checklists such as those mentioned above. Critics of these policy tools point out that values are required to frame, inform, and guide life support decisions. These crucial framing features of life support decision tools have not been well studied; however, it is possible that they might adversely affect the processes and outcomes of care.

Well-intentioned interventions always have the potential for unwanted consequences, whether these 
affect a few patients (e.g., thrombolytic therapy for thrombotic strokes causing intracranial bleeding), or most patients (e.g., anti-arrhythmic drugs for acute myocardial infarction such as flecainide, which was linked to more patient deaths than lives saved). Seemingly helpful interventions regarding life support preferences can also have adverse sequelae. For example, in one US hospital, the consequences of introducing a medical limitation form designed to enhance patient-clinician communication about cardiopulmunary resuscitation (CPR) preferences were analyzed in a qualitative study. ${ }^{27}$ Investigators found that resuscitation discussions became intervention-specific, focusing exclusively on a series of questions about basic and advanced life support, due to the task-oriented prompts of the limitation of care form. Families shared that after trying to close the discussion, physicians continued to describe each technical intervention in detail, leading families to believe that they were neither heard nor trusted.

Policy tools such as an administrative form meant to enhance end-of-life care might inhibit open, respectful communication. ${ }^{27}$ This underscores the need for us to not only develop interventions designed to improve the dying process, but also to conduct local research to ensure that when they are actually implemented in practice, that they do not do more harm than good from the patient and family's point of view.

\section{7) Educate future clinicians}

Recently, medical education research has identified how physicians-in-training interpret patient choices about end-of-life care, how residents can improve their interviewing techniques and communication skills, and how residents may be best prepared for future end-of-life care in the ICU. In university affiliated ICUs participating in the level of care study, we found that half of the CPR directives established in the first $24 \mathrm{hr}$ of ICU admission were established by residents, and affirmed by attending physicians. ${ }^{28}$ This may reflect the fact that in teaching institutions, residents are more often in the hospital than attending physicians. The apprenticeship model of learning underscores that we have much to achieve in practice and medical education to improve future end-of-life care.

There is no question that excellent skills are necessary for physicians of today and tomorrow to aid in the administration, withholding and withdrawal of life support. In a self-administered multicentre survey of ICU residents before and after their ICU rotation, we found that confidence in withdrawal of life support discussions was primarily associated with resident involvement in family meetings. ${ }^{29}$ Residents recom- mended experiential, case-based, patient-centered curricula. They were clear on their need for hands-on learning of the practical and ethical aspects of life support withdrawal, in preference to learning from lectures and textbooks. Residents requested to observe expert role models, and to proceed gradually toward individually supervised responsibility. Among critical care fellows, baseline knowledge of palliative care may be low, and responsive to specific training. ${ }^{30}$

A recent international study has documented that factors associated with decisions to withdraw mechanical ventilation include the need for inotropes, the physicians' prediction of patient's mortality and patient's cognitive function, and the patient's preferences to limit life support. ${ }^{31}$ How clinicians learn to make these projections, and how accurate they become with training and experience are questions that need to be addressed. In addition, future studies to improve the end-of-life education for clinicians would ideally test interactive educational approaches which have been shown to most effectively change clinician behaviour in other settings. ${ }^{32}$ These initiatives should be designed to improve end-of-life care in any hospital setting, not just in the ICU.

\section{8) Personally engage in end-of-life care}

Efforts to provide a 'good death' for patients and their families require close attention to not just their informational and decision-making needs, but also their emotional, spiritual and psychological needs. To this end, clinicians can do their own informal field research. Field notes to record meaningful conversations with patients or family members can help us to be cognizant of the impact of what we say and do. If we ask family members questions such as 'how can we best help you at this difficult time?', some intimacy is shared. With that intimacy can come compelling ideas about ways to improve end-of-life care.

We should be asking grieving family members for feedback on their experience; this may be particularly important in teaching institutions, where families interact with many clinicians-in-training. Consider these four elements that will help residents about to attend a family conference. These suggestions were made to one of us by a family member, the son of a patient who died of disseminated lung cancer. $\mathrm{He}$ asked that we tell our residents this: "Please don't rush. Make me feel like I am the most important person in the world to you right now. Listen twice as much as you talk. Don't just use your mouth, show me how you feel with your eyes."

Some clinicians keep a personal diary of their own end-of-life clinical experiences, which can serve as a 
road map for their personal journey of self-discovery. Akin to field notes in qualitative studies, diaries can be therapeutic narrative tools that generate important insights for us to improve our understanding of ourselves. Some ICUs hold formal multidisciplinary rounds to debrief each death in the ICU. Informal interchanges like this can go a long way to help us understand different perspectives on the nature of the dying process and help health care teams deal with their own bereavement. While we are beginning to address family bereavement in the ICU, we have been slow to recognize that the ICU staff also deal with challenging emotional reactions. Through being in touch with ourselves, we may better reach out to the patients and families we care for. We need to remember that "The success of intensive care is not, therefore to be measured only by the statistics of survival, as though each death were a medical failure. It is to be measured by the quality of lives preserved or restored; and by the quality of the dying of those in whose interest it is to die; and by the quality of human relationships involved in each death." 33

\section{Conclusion}

We should remember that in the ICU, "The time for decisions about life prolonging treatment is a time marked by great uncertainty, by stress and distress, by anguish, and by the threats of impending loss. Preparing for these decisions, making them, and compassionate caring for the sick and for their families involved in these decisions and in the aftermath of these decisions are all integral elements of palliative care. If the ICU is so often the place where such decisions have to be made and lived through, then the ICU is a place for palliative care." ${ }^{34}$

Addressing the needs of dying critically ill patients should be a priority for ICU clinicians. Strategies cited here can be implemented in practice (e.g., determining what dying patients and their families need, personally engaging in end-of-life care, and initiating quality improvement but using quality tools with caution); others are focused on teaching (e.g., educating future clinicians), investigating (e.g., legitimizing research in end-of-life care), and advocating (e.g., promoting social change through professional initiatives). Increasing recognition of our responsibility to provide high quality end-of-life care underpins numerous emerging interventions and initiatives that may ease the dying process for both patients and their families. While we still require more research to understand this process, future studies should include more formal evaluation of the efficacy of these interventions to help point the way to consistent and high quality care for all dying ICU patients.

\section{References}

1 Prendergast TJ, Classens MT, Luce JM. A national survey of end-of-life care for critically ill patients. Am J Resp Crit Care Med 1998; 158: 1163-7.

2 Anonymous. A controlled trial to improve care for seriously ill hospitalized patients. The study to understand prognoses and preferences for outcomes and risks of treatments (SUPPORT). The SUPPORT Principal Investigators. JAMA 1995; 274: 1591-8.

3 Patel R, Sinuff T, Cook D. Influencing advance directive completion rates in nonterminally ill patients: a systematic review. Crit Care 2003; 7(suppl 2): 254 (abstract).

4 Asch DA, Christakis NA. Why do some physicians prefer to withdraw some forms of life support over others? Intrinsic attributes of life-sustaining treatments are associated with physicians' preferences. Med Care 1996; 34: 103-11.

5 Asch DA, Faber-Langendoen K, Shea JA, Christakis $N A$. The sequence of withdrawing life-sustaining treatment from patients. Am J Med 1999; 107: 153-6.

6 Azoulay E, Pochard F, Chevret S, et al. Impact of a family information leaflet on effectiveness of information provided to family members of intensive care unit patients. A multicenter, prospective, randomized, controlled trial. Am J Respir Crit Care Med 2002; 165: 438-42.

7 Schneiderman LJ, Gilmer T, Teetzel HD. Impact of ethics consultations in the intensive care setting: a randomized, controlled trial. Crit Care Med 2000; 28: 3920-4.

8 Consensus statement regarding futile and other possibly inadvisable treatments. Crit Care Med 1997; 25: 887-91.

9 American Thoracic Society. Withholding and withdrawing life-sustaining therapy. Am Rev Respir Dis 1991; 144: 726-31.

10 Rocker G, Dunbar S, for the Canadian Critical Care Society. Withholding or Withdrawal of Life Support: the Canadian Critical Care Society Position Paper. J Palliat Care 2000 S53-S62.

11 Danis M, Federman D, Fins JJ, et al. Incorporating palliative care into critical care education: principles, challenges, and opportunities. Crit Care Med 1999; 27: 2005-13.

12 Curtis JR, Rubenfeld GD. Managing Death in the ICU. The Transition From Cure to Comfort. New York: Oxford University Press; 2001.

13 Rubenfeld GD, Curtis JR. End-of-life care in the intensive care unit: a research agenda. Crit Care Med 2001; 29: 2001-6.

14 Rocker G, Heyland D. New research initiatives in Canada for end-of-life and palliative care. Can Med Assoc J 2003; 169: 300-1. 
15 Curtis JR, Wenrich MD, Carline JD, Shannon SE, Ambrozy DM, Ramsey PG. Patients' perspectives on physician skill in end-of-life care. Differences between patients with COPD, cancer, and AIDS. Chest 2002; 122: 356-62.

16 Nelson JE, Meier DE, Oei EJ, et al. Self-reported symptom experience of critically ill cancer patients receiving intensive care. Crit Care Med 2001; 29: 277-82.

17 Jones C, Griffiths RD, Humphris G, Skirrow PM. Memory, delusions, and the development of acute posttraumatic stress disorder-related symptoms after intensive care. Crit Care Med 2001; 29: 573-80.

18 Heyland DK, Rocker GM, O'Callaghan CJ, Dodek PM, Cook DJ. Dying in the ICU. Perspectives of family members. Chest 2003; 124: 392-7.

19 Curtis JR, Patrick DL, Engelberg RA, Norris K, Asp C, Byock $I$. A measure of the quality of dying and death: initial validation using after-death interviews with family members. J Pain Symptom Manage 2002; 24: 17-31.

20 Pochard F, Azoulay E, Chevret S, et al. Symptoms of anxiety and depression in family members of intensive care unit patients: ethical hypothesis regarding decision-making capacity. Crit Care Med 2001; 29: 1893-7.

21 Azoulay E, Pochard F, Chevret S, et al. Meeting the needs of intensive care unit patient families. A multicenter study. Am J Respir Crit Care Med 2001; 163: 135-9.

22 Heyland DK, Rocker GM, Dodek PM, et al. Family satisfaction with care in the intensive care unit: results of a multiple center study. Crit Care Med 2002; 30: 1413-8.

23 Heyland DK, Cook DJ, Rocker GM, et al. Decisionmaking in the ICU: perspectives of the substitute decision-maker. Intensive Care Med 2003; 29: 75-82.

24 Hall RI, Rocker GM. End-of-life care in the ICU. Treatment provided when life support was or was not withdrawn. Chest 2000; 118: 1424-30.

25 Hall RI, Rocker GM. Can practice variability surrounding end of life care in the ICU be improved? Can J Anesth 2001; 48: A19 (abstract).

26 Grimshaw JM, Shirran L, Thomas R, et al. Changing provider behavior. An overview of systematic reviews of interventions. Med Care 2001; 39(Suppl 2): II2-II45.

27 Ventres W, Nichter $M$, Reed R, Frankel R. Limitation of medical care: an ethnographic analysis. J Clin Ethics 1993; 4: 134-45.

28 Cook DJ, Guyatt G, Rocker G, et al. Cardiopulmonary resuscitation directives on admission to intensive-care unit: an international observational study. Lancet 2001; 358: 1941-5.

29 Stevens L, Cook D, Guyatt G, Griffith L, Walter S, McMullin J. Education, ethics, and end-of-life deci- sions in the intensive care unit. Crit Care Med 2002; 30: 290-6.

30 DeVita MA, Arnold RM, Barnard D. Teaching palliative care to critical care medicine trainees. Crit Care Med 2003; 31: 1257-62.

31 Cook D, Rocker G, Marshall J, et al. Withdrawal of mechanical ventilation in anticipation of death in the intensive care unit. N Engl J Med 2003; 349: 1123-32.

32 Davis D, Thompson O'Brien MA, Freemantle N, Wolf FM, Mazmanian P, Taylor-Vaisey A. Impact of formal continuing medical education. Do conferences, workshops, rounds, and other traditional continuing education activities change physician behavior or health care outcomes? JAMA 1999; 282: 867-74.

33 Dunstan GR. Hard questions in intensive care. A moralist answers questions put to him at a meeting of the Intensive Care Society, Autumn, 1984. Anaesthesia 1985; 40: 479-82.

34 Roy DJ. The times and places of palliative care (Editorial). J Palliat Care 2000; 16(suppl): S3-S4. 